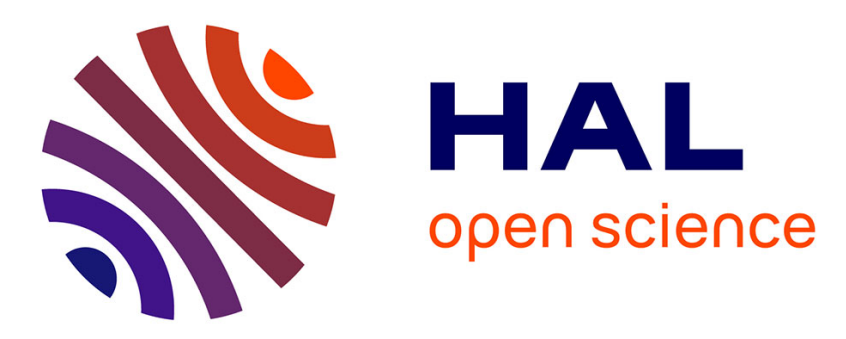

\title{
Calibration of a fully-constrained parallel cable-driven robot
}

Julien Alexandre Dit Sandretto, David Daney, Marc Gouttefarde, Cédric

Baradat

\section{- To cite this version:}

Julien Alexandre Dit Sandretto, David Daney, Marc Gouttefarde, Cédric Baradat. Calibration of a fully-constrained parallel cable-driven robot. [Research Report] RR-7879, INRIA. 2012, pp.21. hal00668921

\section{HAL Id: hal-00668921 \\ https://hal.inria.fr/hal-00668921}

Submitted on 10 Feb 2012

HAL is a multi-disciplinary open access archive for the deposit and dissemination of scientific research documents, whether they are published or not. The documents may come from teaching and research institutions in France or abroad, or from public or private research centers.
L'archive ouverte pluridisciplinaire HAL, est destinée au dépôt et à la diffusion de documents scientifiques de niveau recherche, publiés ou non, émanant des établissements d'enseignement et de recherche français ou étrangers, des laboratoires publics ou privés. 


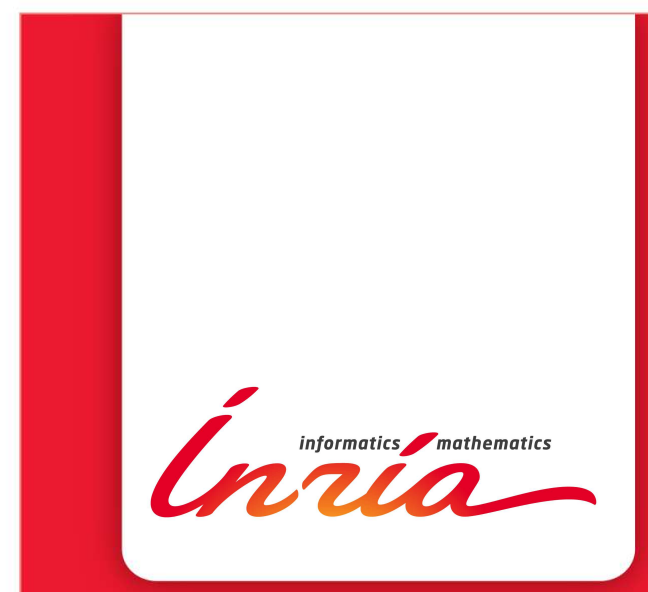

Calibration of a fully-constrained parallel cable-driven robot

Julien Alexandre dit Sandretto

David Daney

Marc Gouttefarde

Cedric Baradat

RESEARCH

REPORT

$\mathbf{N}^{\circ} \mathbf{7 8 7 9}$

Janvier 2012

Project-Teams COPRIN 



\title{
inzián
}

\section{Calibration of a fully-constrained parallel cable-driven robot}

\author{
Julien Alexandre dit Sandretto* \\ David Daney ${ }^{\dagger}$ \\ Marc Gouttefarde $\ddagger$ \\ Cedric Baradat ${ }^{\S}$ \\ Project-Teams COPRIN
}

Research Report $\mathrm{n}^{\circ} 7879$ - Janvier 2012 - 18 pages

\begin{abstract}
An identification of the model parameters for a parallel cable-driven robot is performed by using both a calibration and a self-calibration approach. The manipulator studied is based on a parallel architecture having 8 cables to control the 6 degrees of freedom of its mobile platform so that the mobile platform is fully constrained by the cables. Under some hypotheses on cable properties, the interest of redundancy in actuation is exploited to self-calibrate by using proprioceptive sensors. This approach is compared to the difficulties to implement a calibration process. Additionally, advanced tools and algorithmic improvements are presented to perform the parameter identification. A complete experimentation validates the robot accuracy improvement after calibration or self-calibration. We show that the basic hypotheses on cable properties are verified. Moreover, the investment in terms of time and cost to obtain the external measurements for calibration process does not bring better results and does not balance the simplicity and efficiency of the self calibration process.
\end{abstract}

Key-words: Calibration, Cable-driven Robot, kinematics
* Inria
$\dagger$ Inria
$\ddagger$ Lirmm
$\S$ Tecnalia 


\section{Etalonnage d'un robot parallèle à câbles sur-contraint rapport de recherche Inria}

Résumé : Ce document présente les résultats d'un étalonnage et d'un autoétalonnage d'un robot parallèle à câbles. De plus, certaines méthodes d'identification sont comparées et une nouvelle approche dite globale est décrite.

Mots-clés : Etalonnage, Robot à câbles, Cinématique 


\section{Contents}

1 Introduction 4

2 Cable-driven robot 5

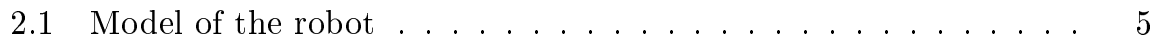

2.1.1 Kinematic relationship ................. 5

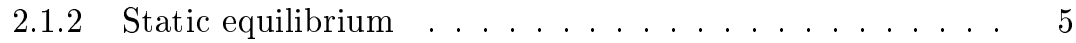

2.1.3 Forward Kinematics ... . . . . . . . . . . . 6

2.1.4 Discussion on inverse kinematic Jacobian . . . . . . . 6

2.2 Redundancy in actuation . . . . . . . . . . . . . 7

3 Calibration $\quad 8$

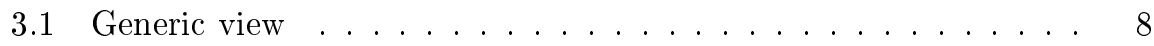

3.1.1 Weighted Least Squares .............. 9

3.1.2 Orthogonal Distance Regression . . . . . . . . . . 9

$3.1 .3 \mathrm{X} 2 \ldots \ldots \ldots \ldots$

3.1.4 Non linear Least Squares solving outlines . . . . . . . . . 9

3.2 Cable-driven robot case ................... . . . 9

3.2.1 Calibration with external measures . . . . . . . . . . . 10

3.2.2 Self-calibration without external measures . . . . . . . 10

4 Experiments $\quad 11$

4.1 Description of the prototype ...................... 11

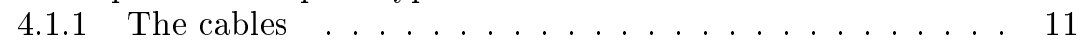

4.2 Model and tools . . . . . . . . . . . . . . . . . . . 12

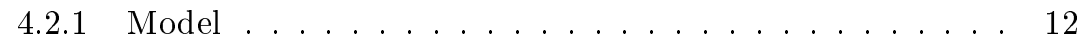

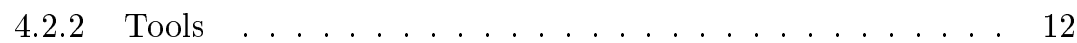

4.3 Measurement . . . . . . . . . . . . . . . . . 12

4.3.1 Acquisition process ...................... 12

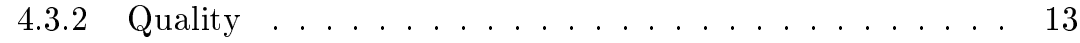

4.4 Results ........................ 13

4.4.1 Parameter identifiability . . . . . . . . . . . 13

4.4.2 Calibration with external measures . . . . . . . . . . 13

4.4.3 Self-calibration without external measures . . . . . . . . 14

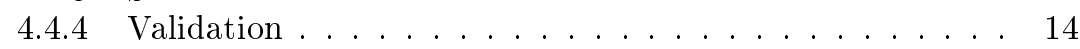

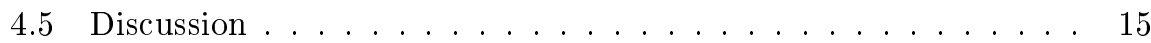

5 Conclusions and future works $\mathbf{1 6}$

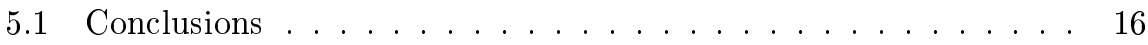

5.2 Future works ..................... 16

6 Acknowledgments $\quad 16$ 


\section{Introduction}

The capabilities of parallel robots explain their use in the industrial applications, from high-speed pick-and-place to precise surgery [14].

Because of tolerances in manufacturing or assembly, the geometry of the actual manipulator does not correspond to the desired design and its theoretical kinematic model. Consequently, the performances of the manipulator, such as its accuracy, are reduced if not lost. This problem could be bypassed by improving the theoretical kinematic model, i.e., by finding the actual values of the kinematic parameters. These parameters, defining the geometry of the robot (frame and platform) and actuator parts, are provided by a kinematic calibration procedure. Calibration consists in identifying model parameters through redundant information on the state of the robot provided by measurements. The difficulties of calibration are both practical and computational. An experimental plan determines the type of redundant information and the way to obtain them, in order to improve the numerical identification of the kinematic parameters computed in a next step.

The more simple and common approach to calibrate a parallel robot is the inverse method as presented in $[22,24]$ which uses the measurements of the pose coordinates, obtained by a theodolite [23] or a camera [17]. This redundant information can be obtained by other extra sensors such as inclinometers [3] or any type of geometrical constraints [8]. In the self-calibration case, the necessary data are provided by additional internal sensor(s). Many solutions have been proposed for parallel manipulators, and some of them [16, 19] may be easily adapted to the case dealt with in the present paper. Indeed, in [16, 19], a length measuring device used as an additional segment that links the base and the mobile of a Gough platform is considered.

This paper focuses on a fully-constrained parallel cable-driven robot. Cabledriven robots have several interesting properties like reduced mass of moving parts (for cables of negligible mass), ease of reconfiguration and, especially, a potentially very large workspace. They are notably used for a flying camera system [1], and have been proposed for heavy loads transportation, for orienting heavy devices and for contour crafting [6]. The cost of these advantages is a complex kinematic and dynamic behavior due to the flexibility, mass and elasticity of the cables. In order to fully constrain the mobile platform, the number of cables must be greater than the number of DOF [15]. In such redundantly actuated cable-driven robots, a high stiffness is generally needed. In our case, the robot is actuated by eight cables for six DOF. Several studies have been run on cable-driven robot kinematics, e.g. [15, 12], but few concerning their calibration. A kinematic calibration based on surface touching or with joint sensors have been reported in [18, 7] and in [20] respectively, whereas a self-calibration procedure for a planar robot is introduced in [5].

As mentioned before, collected information provide redundant equations used for parameter identification. A lot of computational methods have been developed. The basic one is the non linear least squares approach, which computes the parameters so as to match model estimations with measures. Similar methods are orthogonal distance regression (ODR) [4] and $\chi^{2}$ used by Patel in [16]. Different approaches have been proposed like filtering adapted by Wampler in [21], or an original interval approach proposed by Daney and al in [10].

In this paper, kinematics and redundancy of the cable-driven robots are studied. We propose a simplified and robust method for self-calibration and compare it with a more standard "inverse" calibration. Then, we test through experiments the different parameters identification methods based on non linear 
least squares approach. Finally, the results obtained in the case of the studied redundant cable-driven manipulator are then discussed.

\section{Cable-driven robot}

This study is part of a project called CoGiRo (Control of Giant Robot) which notably aims at designing a parallel cable robot having $n=6$ degrees of freedom and a very large workspace. In this paper, a small scale prototype of this parallel cable robot is studied. It uses $m=8$ cables controlling the 6 DOF motion of its mobile platform and its geometry has been chosen so that the platform is fully constrained by the cables.

\subsection{Model of the robot}

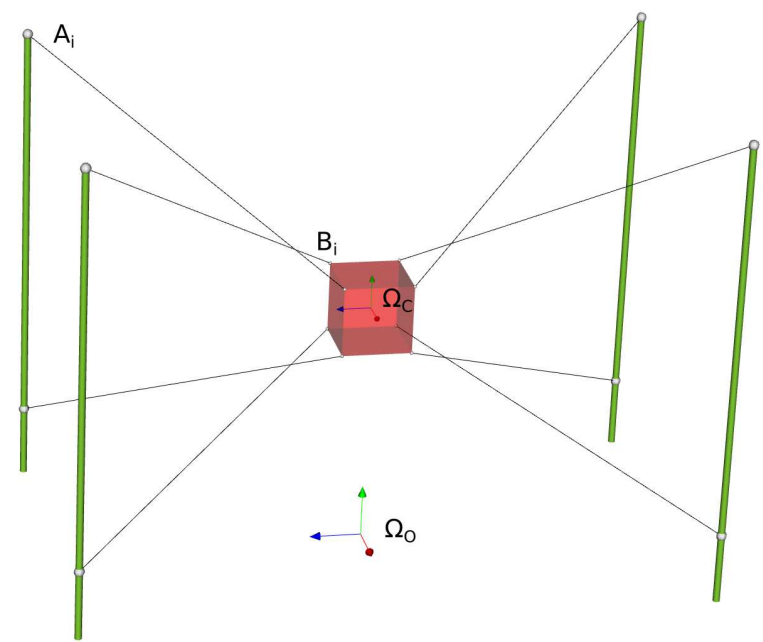

Figure 1: A cable-driven robot

The moving platform or end-effector (mobile reference frame $\Omega_{C}$ ) is connected to the base (fixed reference frame $\Omega_{O}$ ) by $m=8$ cables $(m>n$ to be fully controllable [15]). The $i^{\text {th }}$ cable connects the point $A_{i}$ of the base (coordinate $a_{i}$ in $\Omega_{O}$ ) to the point $B_{i}$ on the mobile platform (coordinate $b_{i}$ in $\Omega_{C}$ ). The pose of the mobile (defined by the position $\mathrm{P}$ and the orientation $\mathrm{R}$ of $\Omega_{C}$ expressed in $\Omega_{O}$ ) is directly controlled by the length and the tension of each cable.

\subsubsection{Kinematic relationship}

The implicit kinematic system of equations is given by

$$
\left\|P+R b_{i}-a_{i}\right\|^{2}-L_{i}^{2}=0, \quad i=[1 \ldots m]
$$

where $L_{i}$ is the distance $A_{i} B_{i}$.

\subsubsection{Static equilibrium}

The static equilibrium is given by a 6 -dimensional equation system :

$$
W \cdot \tau=F
$$


where $\tau=\left[\tau_{1}, \ldots, \tau_{m}\right]$ is the vector of cable tensions, $\mathrm{F}$ is the vector of wrenches (combination of forces and moments) applied on the platform and $W=$ $-J^{-T}, J^{-1}$ being the so-called inverse kinematics Jacobian matrix, function of the pose and the geometrical parameters of the robot [14].

\subsubsection{Forward Kinematics}

The Forward Kinematics FK consists in the solving of eq.1 so as to determine the position and orientation for a given set of cable lengths $L_{i}$. In the case where $m=n$, there are some elaborated solutions [14] which all provide exact solutions. For our cable-driven robot where $m>n$, an exact Forward Kinematics consists in solving an over-constrained system of equations which usually hasn't a solution for a random set of cable lengths. In fact, there are some constraints with a dimension equal to $m-n$ linking the geometry of the robot with the set of joint coordinates, information that can be used to self-calibrate the robot.

In a calibration process, the kinematic parameters are not well estimated and the cable lengths are known up to measurement errors. In this case, the use of a FK can generate errors. An exact solution of a well constraint FK could be found in Complex. One solution is to use a FK based on a Newton-Raphson scheme $\left(F K_{N R}\right)$ with $m=n$ but its convergence with wrong-estimated data is not certain. If $m>n$, an approximated FK (named $F K_{L S}$ ) is based on a non-linear least squares algorithm: the residual error provides an index based on the quality of knowledge of kinematic parameters. However, this scheme could converge to a local minimum therefore providing wrong information in the identification process.

\subsubsection{Discussion on inverse kinematic Jacobian}

During experimentation, we remarked a difference between the inverse kinematic jacobian computed with finite differences and the inverse kinematic [14]. Against all expectations, the finite differences jacobian gives better results, in terms of number of iterations and precision, during the Newton-Raphson scheme used for solving the direct kinematic problem. We use the following vector in order to represent the position and the orientations: $X=[x, y, z, \alpha \mathbf{v}]$, the orientation vector $\delta=\alpha v$ is done with $\mathrm{v}$ the axis vector and $\alpha$ the rotation angle. We know that

$$
\Omega=\dot{\alpha} \mathbf{v}+\sin \alpha \dot{\mathbf{v}}+(1-\cos \alpha) \mathbf{v} \times \dot{\mathbf{v}}
$$

We usually make a one order Taylor approximation with $\alpha$ small :

$$
\Omega \approx \dot{\alpha} \mathbf{v}+\alpha \dot{\mathbf{v}}=\dot{\delta}
$$

Proposition 1 The first order Taylor approximation is too strong for the cosinus, we propose a second order : $\cos \alpha \approx 1-\frac{\alpha^{2}}{2}$ and $\sin \alpha \approx \alpha$

The approximation of $\Omega$ comes :

$$
\begin{aligned}
& \Omega_{2}=\dot{\alpha} \mathbf{v}+\alpha \dot{\mathbf{v}}+\left(1-\left(1-\frac{\alpha^{2}}{2}\right)\right) \mathbf{v} \times \dot{\mathbf{v}} \\
& \text { If we develop : } \\
& \Omega_{2}=\dot{\delta}+\frac{\alpha^{2}}{2} \mathbf{v} \times \dot{\mathbf{v}}=\dot{\delta}+\frac{1}{2} \cdot \alpha \mathbf{v} \times \alpha \dot{\mathbf{v}} \\
& \text { We have } \dot{\delta}=\dot{\alpha} \mathbf{v}+\alpha \dot{\mathbf{v}} \text { and so } \alpha \dot{\mathbf{v}}=\dot{\delta}-\dot{\alpha} \mathbf{v} ; \\
& \Omega_{2}=\dot{\delta}+\frac{1}{2} \cdot \alpha \mathbf{v} \times(\dot{\delta}-\dot{\alpha} \mathbf{v})=\dot{\delta}+\frac{\alpha}{2} \cdot \mathbf{v} \times \dot{\delta} \text { due to } \mathbf{v} \times \mathbf{v}=0
\end{aligned}
$$

Proposition 2 An approximation in order 3 or more needs an estimation of the derivative of angle, and the gain is not significative. 


\begin{tabular}{|c|c|c|c|}
\hline Jacobian & Finite differences & First order & Second order \\
\hline Number of iterations & 2 & 8 & 5 \\
\hline Precision & $10 \mathrm{e}-7$ & $10 \mathrm{e}-5$ & $10 \mathrm{e}-6$ \\
\hline Time elapsed & $7 \mathrm{~ms}$ & $5 \mathrm{~ms}$ & $4 \mathrm{~ms}$ \\
\hline
\end{tabular}

Figure 2: Results medium in some resolution of direct kinematics

Proposition 3 The classical inverse kinematic jacobian $J_{i n v}=\left[n_{i}, n_{i} \times B_{i} C\right]$ could be rewritten as :

$$
\begin{aligned}
\dot{\lambda_{i}} & =\left[n_{i}, n_{i} \times B_{i} C\right] \cdot \dot{X}+\left[n_{i}, n i \times B_{i} C\right] \cdot\left(\mathbf{0}, \frac{\alpha}{2} \mathbf{v} \times \dot{\delta}\right) \\
& =\left[n_{i}, n_{i} \times B_{i} C\right] \cdot \dot{X}+\left[n_{i},\left(n i \times B_{i} C\right) \times \frac{\alpha}{2} \mathbf{v}\right] \cdot(\mathbf{0}, \dot{\delta}) \\
& =\left[n_{i}, n_{i} \times B_{i} C+\left(n_{i} \times B_{i} C\right) \times \frac{\alpha}{2} \mathbf{v}\right] \cdot \dot{X}
\end{aligned}
$$

And so, $J_{\text {inv }}$ comes

$$
J_{i n v}=\left[n_{i}, n_{i} \times B_{i} C+\left(n_{i} \times B_{i} C\right) \times \frac{\alpha}{2} \mathbf{v}\right]
$$

Or if we prefer to keep the classical Plucker line coordinates Jacobian, we could write :

$$
J_{\text {inv } 2}=J_{i n v} \cdot(I+M)
$$

where $M$ is the pre cross product of $\frac{\alpha}{2} \mathbf{v}$.

Results in simulation The corrected term $\frac{\alpha}{2} \cdot \mathbf{v} \times\left(n_{i} \times B_{i} C\right)$ has attained sometimes 0.1 radian, enough to gain 6 iterations and a 100 factor in the precision of the FK.

Results in number of iterations, time and precision in forward kinematics scheme are presented in Table 2.1.4. We have also good results in selfcalibration, in this case, the position are not known and the error could be important, this new jacobian is so very usefull.

To conclude, a forward kinematic scheme using this corrective term gives better and faster solution. The self-calibration procedure gains lot of time and in some case converge when the classical Jacobian didn't allow it.

\subsection{Redundancy in actuation}

As defined in [11, 14], the cable robot studied in this paper is redundant in terms of actuation because the end-effector motion is over-constrained by the actuators. One question is to define how this redundancy can be used to provide redundant information on the state of the robot.

If we consider the elasticity and the mass of the cables, the lengths depend on the cable tensions and on the pose of the robot. This is modeled by $m$ additional equations $L_{i}=\mathcal{L}\left(\rho_{i}, \tau_{i}\right)$ with $\rho_{i}$ the articular coordinate (length of the cable $i$ unwound by a drum). As a consequence, the kinematic and the static model eqs(2) and (1) are linked: the number of equations for one configuration of the robot is equal to $m$ in eq.1 and 6 in eq(2). If a self-calibration problem is considered, the 6 pose coordinates $(P$ and $R$ ) are unknown. For a known value 
of the external force $F$ the set of $m$ variables $\tau_{i}$ have to be taken in account. A self-calibration is possible only when information even partial are given about the tensions $\tau_{i}$.

If we suppose that the elasticity and the mass of the cables are negligible, the kinematic and the static relationships are then independent, and can be solved separately. In other words, the 6 pose coordinates (position and orientation) of the mobile platform should be deduced from the $m=8$ kinematic relations of eq(1). They are simply functions of geometrical parameters $\xi=\left[a_{1 . . m}, b_{1 . . m}, \Delta l_{1 . . m}\right]$ and controlled cable lengths $\rho_{1 . . m}$. We have a direct relation $L_{i}=\rho_{i}+\Delta l_{i}$, where $\Delta l_{i}$ is the offset length in the $i^{\text {th }}$ cable $(i=1 . . m)$. This system is now over-constrained and gives two equations (after pose coordinate elimination) that link the internal measurement $\rho_{i}$ and the kinematic parameters $\xi$. This hypothesis on cable properties simplifies the calibration. The validity of this hypothesis can be verified with an Irvine model simulation or a Bouchard test $[13,7]$. We will see, in the following, that the giant crane prototype is in this case.

\section{Calibration}

The calibration goal is to enhance the robot performances by improvement of model knowledge. The latter consists in identifying the model parameters through redundant information on the state of the robot provided by measurements.

We will see that calibration can be considered as a generic process [21]. We will make a difference between the case where we have additional external measures on the state of the robot and the case where the proprioceptive sensor data of the robot are sufficient for calibration (also called self-calibration).

The robots studied, $m>n$, are redundant in terms of measurement if we make the hypothesis of non elastic and mass-less cables (see section 2.2).

\section{$3.1 \quad$ Generic view}

Based on [21], for each of the $N_{C}$ measure configuration the calibration equations links three types of variables:

The measurements $M_{k}\left(k=1 . . N_{C}\right)$, the parameters $\xi$ we want to identify (geometrical parameters) and unknowns variables $\Upsilon$ required to model our equations. These variables $\Upsilon=\left[\hat{\Upsilon}, \check{\Upsilon}_{1 \ldots N_{C}}\right]$ should be

- Constant $\hat{\Upsilon}$ : their values do not change during the calibration process;

- Variable as a function of the robot configurations $\check{\Upsilon}_{k=1 . . N_{C}}$.

We consider a system of equations linking a set of measures $M$ and the unknowns $V=[\xi, \Upsilon]$ in the calibration equations:

$$
f_{k}\left(M_{k}, V\right) \simeq 0, \quad k=\left[1 \ldots N_{C}\right]
$$

A solution of the system (7) could be computed by different methods, most of them give a non linear least squares solution which minimizes the criteria $F^{T} . F$ with $F=\left[f_{1}, \ldots, f_{N_{c}}\right]^{T}$. This could be obtained with a classical LevenbergMarquart algorithm but some improvement on criterion definition are possible. 


\subsubsection{Weighted Least Squares}

The WLS technique introduces the ability to prioritize a measure by considering the criterion $F^{T} \Sigma_{F} F$. The weight matrix $\Sigma_{F}$ is built as a function of knowledge on some uncertainties linked with the measurement modeled through a covariance matrix $\Sigma_{M}$. A linear approximation of $\Sigma_{F}$ is obtained as $\Sigma_{F}=J_{M}^{T} \Sigma_{M} J_{M}$ with $J_{M}=\frac{\partial F}{\partial M}$.

\subsubsection{Orthogonal Distance Regression}

ODR takes into account the possible errors in measurements [4] and considers the criteria $F^{T} \Sigma_{F} F+\tilde{M}^{T} \Sigma_{M} \tilde{M}$. We put $\tilde{M}$ as the difference between the current $M$ and the initial $M$.

\subsubsection{X2}

$\chi^{2}$ permits a control of deviation in identification of the unknowns [16]. The considered criteria is now $F^{T} \Sigma_{F} F+\tilde{M}^{T} \Sigma_{M} \tilde{M}+\tilde{V}^{T} \Sigma_{V} \tilde{V}$. We put $\tilde{V}$ as the difference between the current $V$ and the initial $V$.

\subsubsection{Non linear Least Squares solving outlines}

Classical non linear least squares problems solving is based on iterative linear approximation using a Taylor's linearization. Partial differentiation gives the following over-constrained linear system:

$$
J_{V} \cdot \Delta V=\Delta F
$$

with the Jacobian $J_{V}=\frac{\partial f}{\partial V}$ and the condition to obtain an over-constrained system of equations given by:

$$
N_{C} \times \operatorname{dim} f_{k}>\operatorname{dim} \xi+\operatorname{dim} \hat{\Upsilon}+N_{C} \times \operatorname{dim} \check{\Upsilon}_{k}
$$

The system of eq(8) can be solved with an SVD (singular value decomposition) of $J_{V}$.

In order to check if parameters are solvable, the following condition has to be verified [2]:

$$
\begin{aligned}
\operatorname{rank}\left(J_{V}\right) & =\operatorname{dim}(V) \\
& =\operatorname{dim} \xi+\operatorname{dim} \hat{\Upsilon}+N_{C} \times \operatorname{dim} \check{\Upsilon}_{k}
\end{aligned}
$$

\subsection{Cable-driven robot case}

For the parallel cable robot calibration, the equations used are directly the kinematic relationships (1) provided that the hypothesis of negligible cable elasticity and mass (see section 2.2) is acceptable:

$$
\begin{aligned}
f_{k, i}\left(M_{k}, V\right) & =\left\|P_{k}+R_{k} b_{i}-a_{i}\right\|^{2}-\left(\rho_{k, i}+\Delta l_{i}\right)^{2} \\
& =0
\end{aligned}
$$

for $k=1 \ldots N_{C}$ and $i=1 \ldots m$.

Now let's discuss two different calibration approaches, with and without external measurements. 


\subsubsection{Calibration with external measures}

In addition to the articular coordinates given by the proprioceptive sensors, the measurement of the robot pose (position and orientation) provided by an external device like a camera or a laser tracker is assumed to be available. The calibration system to be solved is made of the functions $f_{k, i}\left(M_{k}, V\right)$ with the following data:

- $M_{k}=\left[\rho_{i, k}, P_{k}, R_{k}\right]$

- $\xi=\left[a_{i}, b_{i}, \Delta l_{i}\right]$

- $V=[\Upsilon, \xi]=[\emptyset, \xi]=\xi$

In the experimentation section, we will solve this system with a weighted least squares and an ODR method.

\subsubsection{Self-calibration without external measures}

If we don't have any external measurement, we can calibrate the robot with the proprioceptive sensors only.

The calibration system to be solved is still made of the functions $f_{k, i}\left(M_{k}, V\right)$ but with the following data:

- $M_{k}=\left[\rho_{i, k}\right]$

- $\xi=\left[a_{i}, b_{i}, \Delta l_{i}\right]$

- $V=[\Upsilon, \xi]=\left[P_{k}, R_{k}, \xi\right]$

With these data, the Jacobian $J_{V}$ of eq 8 is composed of the Jacobian of kinematics parameters (as in a calibration case) $J_{\xi}$ and of the inverse kinematics Jacobian $J_{\Upsilon}$.

One difficulty of calibration is to eliminate the $\check{\Upsilon}_{k}=\left[P_{k}, R_{k}\right]$ variables [9] in the identification vector $V=\left[\check{\Upsilon}_{k}, \xi\right]$. In [16], it is done indirectly with an iterative Forward Kinematics in order to determine $\Upsilon$ in each iteration of the identification algorithm. We propose a complete identification which looks for $\Upsilon$ together with $\xi$. This allows us to avoid the problem of the FK convergence.

Hereafter, we compare elimination in the Forward Kinematics and the complete approach.

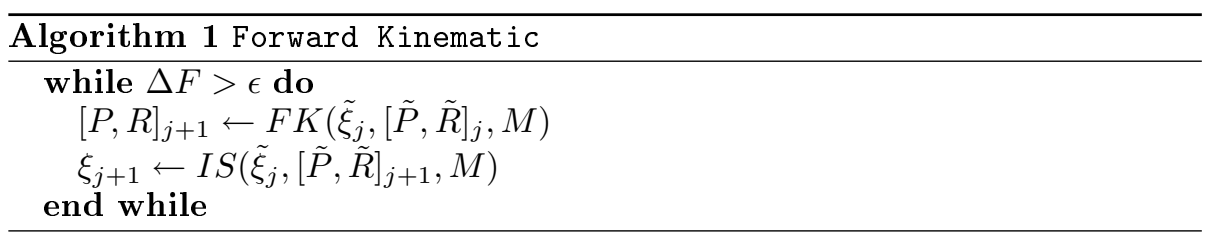

In algorithm 3.2.2, IS is an identification scheme, which actually could be a non linear least square scheme solving the system (8).

The Forward Kinematics (FK) step uses an approximation of the parameters $\xi$ to find the pose of the robot $[P, R]$ for all configurations, the solution is then not established and can be far from the expected position (section 2.1.3). Consequently, an iteration may not improve the calibration but instead deteriorates it. This phenomenon is observed in our case. This is why, we use the following method referred to as a complete identification process. 


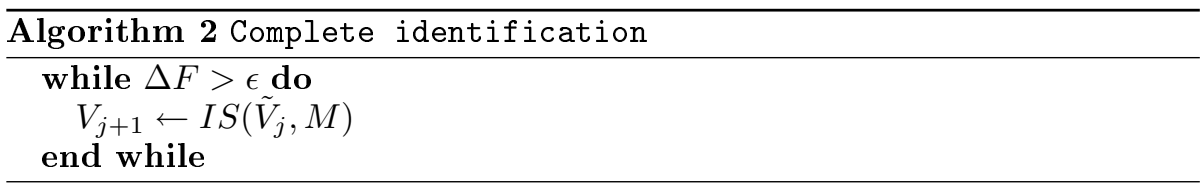

Complete identification uses only an identification scheme, the pose coordinates and the geometrical parameters are computed at the same time and, therefore uncertainty on the pose determination convergence is eliminated.

In the experimentation section, we solve this system with a weighted least squares and a $\chi^{2}$ method.

\section{Experiments}

\subsection{Description of the prototype}

The prototype, shown in Fig. 3, was built by TECNALIA in collaboration with the LIRMM and is used as a small scale prototype of the future large parallel cable robot to be built in CoGiRo project framework.

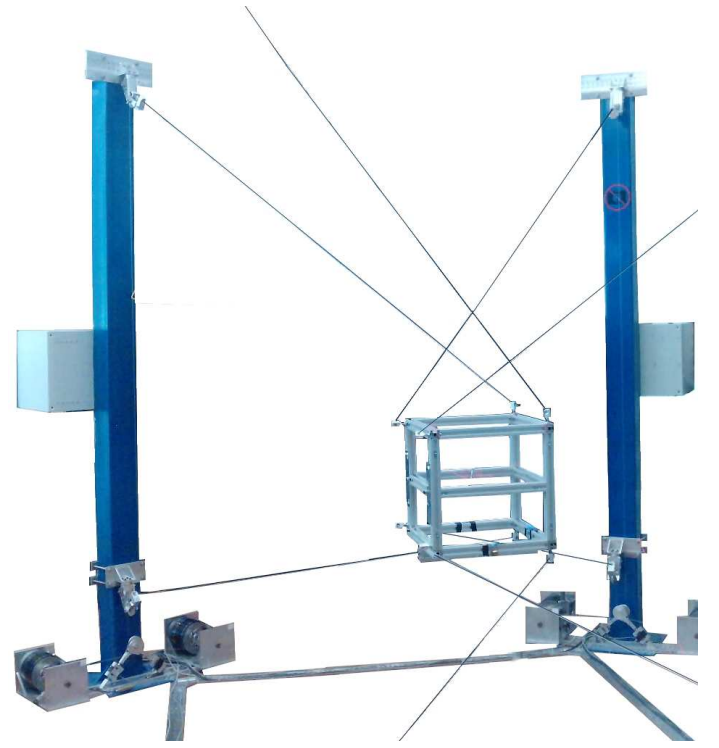

Figure 3: Partial view of the prototype

ReelAx8 is a reconfigurable cable driven robot. Eight cables, wound round winches, are each attached to the eight corners of a cube shaped platform of about 40 centimeters, by means of spherical joints. The winches are fixed 2 by 2 on four posts up to three meters arranged at the four corners of a three by four meters rectangle.

\subsubsection{The cables}

The cables are of galvanized steel with single-strand 1x19 structure and diameter of $1.2 \mathrm{~mm}$.

The design of the actuation system is such that the path length of the cables through the different pulleys is constant from the drum to the eyelet. The drum 
is linked to the motor stator with a prismatic joint (linear bearing and rods), and a ball screw/nut system translates the drum (that way the cable always winds up at the same place on the drum). The eyelet is static and friction of the cable is minimized by pre-orienting the eyelet axis toward the center of the workspace. The actuators are direct drives RTMB0140-070 from Etel Motion Tech.

The actuators include optical incremental encoders with 5000 lines. To measure the tension in the cables, one pulley on each robot axis is equipped with force sensors SM5424 from Sensel Measurement.

The control system is based on xPC Target from Mathworks. In order to minimize the wiring, the drives are decentralized and placed near the motors, force sensors and encoders. They are connected to the control system by means of a real-time field-bus.

\subsection{Model and tools}

In the following paragraph, we describe the model and the tools implemented for the identification of ReelAx8 prototype kinematic parameters.

\subsubsection{Model}

We choose the classical geometrical model of eq(1) without the static equilibrium relationship of eq(2). In fact, we can neglect the elasticity and mass of the cables. This assumption has been verified by computing the possible stretching (below one millimeter) and by Irvine model comparison [7, 13].

\subsubsection{Tools}

We use a toolbox based on GSL (GNU Scientific Library), which contains functions for robotic modeling, different methods for parameters identification (LS, ODR, $\chi^{2}$ ) and a non linear solver based on Levenberg-Marquardt.

For the outliers filtering, an important step described in [22], we have used a filter based on a scaled Gaussian (forced to zero under 3\%) which permits the computation of a good weight for each measure.

In addition, a function allowing the verification of the parameter observability based on a QR decomposition of the identification matrix has been used.

\subsection{Measurement}

The measurements were made by means of a laser tracker system and a portable $3 \mathrm{D}$ measuring arm.

\subsubsection{Acquisition process}

The acquisition of the measurements were made in 2 different steps.

First, we found the estimation of geometrical parameters. We measured the eyelet positions on the frame with the laser tracker and the attachment points on the mobile platform with a portable 3D measuring arm.

The pose measurement step could then start. We placed the mobile platform in 44 different poses and took measures of three types:

- Proprioceptive sensors gave cables lengths ; 
- The robot force sensors gave us the tension in the cables (we don't use these data in this paper);

- Positions of three points measured with the laser gave us the position and orientation of the mobile platform.

Comment: we took the measures on the borders of the workspace [10], but the prototype, due to a non-mature control, didn't provide a large workspace compared with $a_{i}$. In fact, the platform could move about $30 \mathrm{~cm}$ around the center and rotate 5 degrees in three directions.

Now, we are going to see, that this could have been a problem for the identification of all parameters.

\subsubsection{Quality}

A measure is useful if its precision is known. Having this in mind, we estimated the expected error for each device. This error was used in the verification step and in the computation of the weights of the identification process. In our case we considered a mix between the precision of measurement device and the way the acquisition was done(maximized on purpose): $\sigma_{\text {poses }}=5 \mathrm{~mm}$, $\sigma_{\text {length }}=5 \mathrm{~mm}, \sigma_{A}=20 \mathrm{~mm}, \sigma_{B}=10 \mathrm{~mm}, \sigma_{\Delta l}=100 \mathrm{~mm}$.

\subsection{Results}

From the 44 measures done, we used 30 measures for the identification and 10 for validation ( 4 outliers were eliminated).

In our calibration study and with the goal of adapting it on a giant robot, we made an important work investment to obtain well-estimated kinematic parameters (by laser and CMM measurements) in order to check the robustness of the algorithms used.

Each result was judged in terms of convergence, initial and final errors on cable lengths and improvement on the validation measures.

\subsubsection{Parameter identifiability}

Initially, we identified the $\left[a_{i}, b_{i}, \Delta l_{i}\right]_{i=1 . .8}$ but we found a strong dependence between the $a_{i}$ and the $b_{i}$. This came from the small rotations allowed by the prototype. The parameters $a_{i}$ and $b_{i}$ are linked by the relation $R \dot{b}_{i}-a_{i}$ in the identification equations. It's not an important problem, indeed, the parameters of the platform are well known, easy to measure and don't change unlike $\Delta l_{i}$ and $a_{i}$, which changes at each new configuration, restarting of motors, etc. In the particular case of self-calibration, it's necessary to choose the reference frame of the robot as follows [2] :

$a_{1 x}=a_{1 y}=a_{1 z}=a_{2 y}=a_{2 z}=a_{3 z}=0$.

\subsubsection{Calibration with external measures}

The results of the calibration eq.7 (residual error on the cable lengths) are collected in the first part of table 1 .

After 3 iterations, we reach a correct minimum (with a medium error at $3 \mathrm{~mm}$ for WLS and $1 \mathrm{~mm}$ for ODR) and after about 10 iterations the solver stops at the expected precision $\left(\Delta F<10^{-8}\right)$. The convergence region is huge enough that initial parameters are not necessary. 


\begin{tabular}{|c|c|c|c|}
\hline & WLS & \multicolumn{2}{|c|}{ ODR } \\
\hline \multicolumn{4}{|c|}{ Identification ( 30 configurations) } \\
\hline \multirow[t]{2}{*}{ Initial error } & mean(std) & \multicolumn{2}{|c|}{ mean(std) } \\
\hline & $20 \mathrm{~mm}(20 \mathrm{~mm})$ & \multicolumn{2}{|c|}{$20 \mathrm{~mm}(20 \mathrm{~mm})$} \\
\hline \multirow[t]{2}{*}{ Final error } & mean(std) & \multicolumn{2}{|c|}{ mean(std) } \\
\hline & $3 \mathrm{~mm}(6 \mathrm{~mm})$ & \multicolumn{2}{|c|}{$1 \mathrm{~mm}(4 \mathrm{~mm})$} \\
\hline \multicolumn{4}{|c|}{ Validation (10 configurations) } \\
\hline \multirow{2}{*}{$\begin{array}{l}\text { Error on calibra- } \\
\text { tion equation }\end{array}$} & initial final & \multicolumn{2}{|c|}{ initial final } \\
\hline & $40 \mathrm{~mm} \quad 6 \mathrm{~mm}$ & $40 \mathrm{~mm}$ & $9 \mathrm{~mm}$ \\
\hline \multirow{2}{*}{$\begin{array}{l}\text { Error on posi- } \\
\text { tioning }\end{array}$} & initial final & \multicolumn{2}{|c|}{ initial final } \\
\hline & $37 \%$ & $37 \%$ & $10 \%$ \\
\hline \multirow{2}{*}{$\begin{array}{l}\text { Error on orient- } \\
\text { ing }\end{array}$} & initial final & \multicolumn{2}{|c|}{ initial final } \\
\hline & \begin{tabular}{|l|l|}
$40.2 \%$ & $25 \%$ \\
\end{tabular} & $40.2 \%$ & $21 \%$ \\
\hline
\end{tabular}

Table 1: Results for the calibration

\subsubsection{Self-calibration without external measures}

The results of the calibration eq.7 (residual error on the cable lengths) are collected in the first part of table 2 .

The unknown parameters $\Upsilon_{k}=[P, R]_{k}$ are initialized with a $F K_{L S}$ process. The convergence is checked for the measurement configurations. Note that, if the kinematic parameter estimation is too rough, $F K_{L S}$ doesn't find acceptable solution and the self-calibration cannot be started.

As for the calibration, after 4 iterations we reach a correct minimum (with a medium error at $0.5 \mathrm{~mm}$ for WLS and $2 \mathrm{~mm}$ for $\chi^{2}$ ) and after about 10 iterations the solver stops at the expected precision $\left(\Delta F<10^{-8}\right)$.

\subsubsection{Validation}

First, a simple validation is done by the checking of model improvement on the 10 validation measures. The results (residual error on the cable lengths) are then presented in the second part of table 1 for calibration and table 2 for self-calibration.

Second, we validate the manipulator accuracy by computing the improvement of general positioning. We measure a moving (difference) between two validation configurations, on position $\Delta P_{\text {meas }}$, and on orientation $\Delta R_{\text {meas }}$ (in Euler angles). We compute the theoretical moving $\Delta P_{\lambda}$ and the rotation $\Delta R_{\lambda}$ with a FK process, where $\lambda$ means the kind of kinematic parameters used in the FK (i.e initial, post WLS calibration, post ODR, post WLS self-calibration, or post $\chi^{2}$ identification). The results are given as relative error in percentages and defined by $100 *\left(\frac{\Delta P_{\lambda}-\Delta P_{\text {meas }}}{\Delta P_{\text {meas }}}\right)$.

A graphical result for one displacement is shown in detail in fig. 4 for the positioning and fig. 5 for the orienting.

The complete results are expressed with a maximal error on $P$ and $R$ given in percentages. They are shown in the lower part of table 1 for calibration and table 2 for self-calibration.

We see that the orienting is not perfectly corrected (except for the $\chi^{2}$ method), probably because we could not obtain measurements in a wide workspace 


\begin{tabular}{|c|c|c|c|c|}
\hline & \multicolumn{2}{|c|}{ WLS } & \multicolumn{2}{|c|}{$\chi^{2}$} \\
\hline \multicolumn{5}{|c|}{ Identification (30 configurations) } \\
\hline \multirow[t]{2}{*}{ Initial error } & \multicolumn{2}{|c|}{ mean(std) } & \multicolumn{2}{|c|}{$\operatorname{mean}($ std $)$} \\
\hline & \multicolumn{2}{|c|}{$20 \mathrm{~mm}(30 \mathrm{~mm})$} & \multicolumn{2}{|c|}{$20 \mathrm{~mm}(30 \mathrm{~mm})$} \\
\hline \multirow[t]{2}{*}{ Final error } & \multicolumn{2}{|c|}{$\operatorname{mean}($ std $)$} & \multicolumn{2}{|c|}{ mean(std) } \\
\hline & \multicolumn{2}{|c|}{$0.5 \mathrm{~mm}(0.5 \mathrm{~mm})$} & \multicolumn{2}{|c|}{$2 \mathrm{~mm}(4 \mathrm{~mm})$} \\
\hline \multicolumn{5}{|c|}{ Validation (10 configurations) } \\
\hline \multirow{2}{*}{$\begin{array}{l}\text { Error on calibra- } \\
\text { tion equation }\end{array}$} & initial & final & initial & final \\
\hline & $40 \mathrm{~mm}$ & $1 \mathrm{~mm}$ & $40 \mathrm{~mm}$ & $0.7 \mathrm{~mm}$ \\
\hline \multirow{2}{*}{$\begin{array}{l}\text { Error on posi- } \\
\text { tioning }\end{array}$} & initial & final & \multicolumn{2}{|c|}{\begin{tabular}{|l|l} 
initial final \\
\end{tabular}} \\
\hline & $37 \%$ & $1.2 \%$ & $37 \%$ & $0.3 \%$ \\
\hline \multirow{2}{*}{$\begin{array}{l}\text { Error on orient- } \\
\text { ing }\end{array}$} & initial & final & \multicolumn{2}{|c|}{ initial final } \\
\hline & $40.2 \%$ & $12 \%$ & $40.2 \%$ & $1.9 \%$ \\
\hline
\end{tabular}

Table 2: Results for the self-calibration

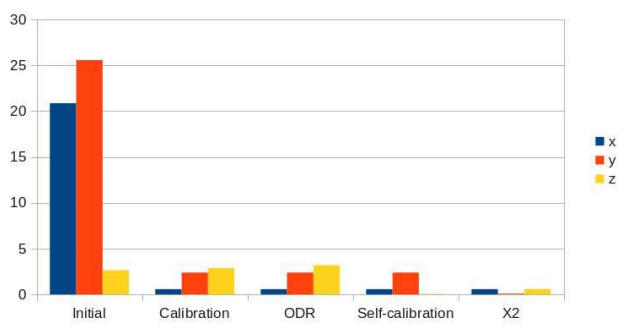

Figure 4: Error on positioning \% (for initial parameters and after 4 different identification methods)

at different orientations larger than 5 degrees.

\subsection{Discussion}

A restricted workspace, with small possible moves in relation with the frame dimension, and small rotation amplitudes (less than 5 degrees) seem to challenge the geometry used for the prototype. The future parallel cable robot architecture will be different in accordance with this remark.

These difficulties led us to calibrate only the position of the $a_{i}$ and the $\Delta l_{i}$. Actually, the geometry of the platform being well known and easy to get, this problem was naturally bypassed.

The algorithms used for the identification are all power-full in term of convergence and residual error. The discussions focus on the results about independent measures of each method, and mainly on the comparison between calibration and self-calibration.

The comparison between calibration with least squares and ODR is not simple, because the parameter identification results are close. It means that the measures with laser tracker have low errors.

In the case of self-calibration, initial parameter estimation is essential because the feasibility relies on it and leads to choose a specific process. In our case, we have a good first estimation and therefore, in addition to a good conver- 


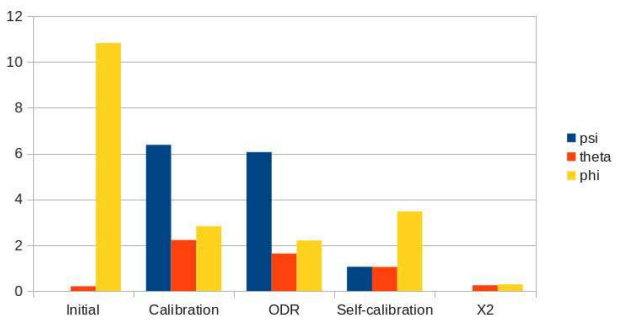

Figure 5: Error on orienting \% (for initial parameters and after 4 different identification methods)

gence, the $\chi^{2}$ provides a deviation control if some parameters are not perfectly identifiable (due to the robot geometry or measurement) as observed in the orientation accuracy. The least squares give also good results.

The real comparison is thus between calibration and self-calibration. In these experiments, we can conclude that self-calibration gives good enough results.

Moreover, the self-calibration is quite cheap, the cable lengths are given by the control with a sufficient accuracy.

On the other hand, with a bad estimation of parameters the calibration process is more robust than self-calibration, which can easily derive, resulting in a bad identification of the robot parameters.

\section{Conclusions and future works}

\subsection{Conclusions}

In this paper, we've verified experimentally the hypothesis of self-calibration capacity for a particular parallel cable-driven robot. Now those allow us to test a simple new approach for the elimination of pose variables in the self-calibration process. We try three different methods derived from the least squares approach for the parameter identification, and make some proposals on their use. To conclude, our robot can be either calibrated if we don't have accurate kinematics parameter estimation, or self-calibrate, both with robust algorithms.

\section{$5.2 \quad$ Future works}

The robot under construction for the CoGiRo project will have a different geometry and different cables to handle heavy loads; for that, a new model is in progress with mass and elasticity consideration. Future works will include certification of the identification results. In the project, we plan to use camera for $3 \mathrm{D}$ pose sensing, and we are developing a calibration method based on the vision result.

\section{Acknowledgments}

This work was supported for one part by the French National Research Agency (ANR) under grant 2009-SEGI-018 (CoGiRo ANR project) and for the other part by the Région Languedoc-Roussillon under grant 115217.

Thanks to Gilles Trombettoni for fruitful discussions. 


\section{References}

[1] Skycam, 2007.

[2] Sebastian Besnard and Wisama Khalil. Identifiable parameters for parallel robots kinematic calibration. In International Conference on Robotics and Automation, volume 3, pages 2859-4729, Seoul, Korea, may 2001.

[3] SÃ C bastien Besnard and Wisama Khalil. Calibration of parallel robots with two inclinometers. In Robotics and Automation, volume 3, pages $1758-1763,1999$.

[4] Paul T. Boggs, Richard H. Byrd, and Robert B. Schnabel. A stable and efficient algorithm for nonlinear orthogonal distance regression. SIAM Journal on Scientific and Statistical Computing, 8(6):1052-1078, 1987.

[5] P.H. Borgstrom, B.L. Jordan, B.J. Borgstrom, M.J. Stealey, G.S. Sukhatme, M.A. Batalin, and W.J. Kaiser. Nims-pl: A cable-driven robot with self-calibration capabilities. Robotics, IEEE Transactions on, 25(5):1005 -1015, oct. 2009.

[6] Paul Bosscher and al. Cable-suspended robotic contour crafting system. Automation in Construction, 17(1):45 - 55, 2007.

[7] Samuel Bouchard. Geometrie des robots paralleles entraines par des cables. $\mathrm{PhD}$ thesis, Universite Laval, Quebec, 2008.

[8] D. Daney. Self calibration of gough platform using leg mobility constraints. In in Proc. 10th World Congress Theory of Machine and Mechanisms, Oulu, Finland, pages $104-109,1999$.

[9] David Daney. Etalonnage geometrique des robots paralleles. PhD thesis, Nice - Sophia Antipolis, 2000.

[10] David Daney, Nicolas Andreff, Gilles Chabert, and Yves Papegay. Interval method for calibration of parallel robots : Vision based experiments. Mechanism and Machine Theory, 41:929-944, 2006.

[11] Pierrot Francois. Parallel mechanisms and redundancy. In 1st Int. Colloquium, Collaborative Research Centre 562, pages 261-277, 2002.

[12] M. Gouttefarde, J.-P. Merlet, and D. Daney. Determination of the wrenchclosure workspace of 6-dof parallel cable-driven mechanisms. In Jadran Lennarcic and B. Roth, editors, Advances in Robot Kinematics, pages 315322. Springer Netherlands, 2006.

[13] H. M. Irvine and T. K. Caughey. The linear theory of free vibrations of a suspended cable. Proceedings of the Royal Society of London. Series A, Mathematical and Physical Sciences, 341(1626):pp. 299-315, 1974.

[14] Jean-Pierre Merlet. Parallel Robots, 2nd Edition, volume 128. Springer, 2006.

[15] A Ming and $T$ Higuchi. Study On Multiple Degree-OfFreedom Positioning Mechanism Using Wires .2. Development Of A Planar Completely Restrained Positioning Mechanism. International Journal Of The Japan Society For Precision Engineering, 28(3):235-242, Sep 1994. 
[16] Amit J. Patel and Kornel F. Ehmann. Calibration of a hexapod machine tool using a redundant leg. International Journal of Machine Tools and Manufacture, 40(4):489-512, mar 2000.

[17] P. Renaud, N. Andreff, P. Martinet, and G. Gogu. Kinematic calibration of parallel mechanisms: A novel approach using legs observation. Robotics, IEEE Transactions on, 21(4):529 - 538, aug. 2005.

[18] S. Tadokoro, R. Verhoeven, M. Hiller, and T. Takamori. A portable parallel manipulator for search and rescue at large-scale urban earthquakes and an identification algorithm for the installation in unstructured environments. In Intelligent Robots and Systems, 1999. IROS '99. Proceedings. 1999 IEEE/RSJ International Conference on, volume 2, pages $1222-1227$ vol.2, 1999 .

[19] Yukio Takeda, Gang Shen, and Hiroaki Funabashi. A DBB-Based kinematic calibration method for In-Parallel actuated mechanisms using a fourier series. Journal of Mechanical Design, 126(5):856-865, 2004.

[20] M. Saeed Varziri and Leila Notash. Kinematic calibration of a wire-actuated parallel robot. Mechanism and Machine Theory, 42(8):960 - 976, 2007.

[21] C. Wampler and T. Arai. Calibration of robots having kinematic closed loops using nonlinear least-squares estimation. Proc. IFToMM-jc Int. Symp. Theory Mach. Mech., pages 153 - 158, 1992.

[22] Charles W. Wampler, John M. Hollerbach, and Tatsuo Arai. An implicit loop method for kinematic calibration and its application to Closed-Chain mechanisms. IEEE Transactions on Robotics and Automation, 11(5):710 724 , oct 1995 .

[23] Hanqi Zhuang, O. Masory, and Jiahua Yan. Kinematic calibration of a stewart platform using pose measurements obtained by a single theodolite. In Intelligent Robots and Systems 95. 'Human Robot Interaction and Cooperative Robots', Proceedings. 1995 IEEE/RSJ International Conference on, volume 2, pages 329 -334 vol.2, aug 1995.

[24] Hanqi Zhuang, Jiahua Yan, and Oren Masory. Calibration of stewart platforms and other parallel manipulators by minimizing inverse kinematic residuals. Journal of Robotic Systems, 15(7):395-405, 1998. 


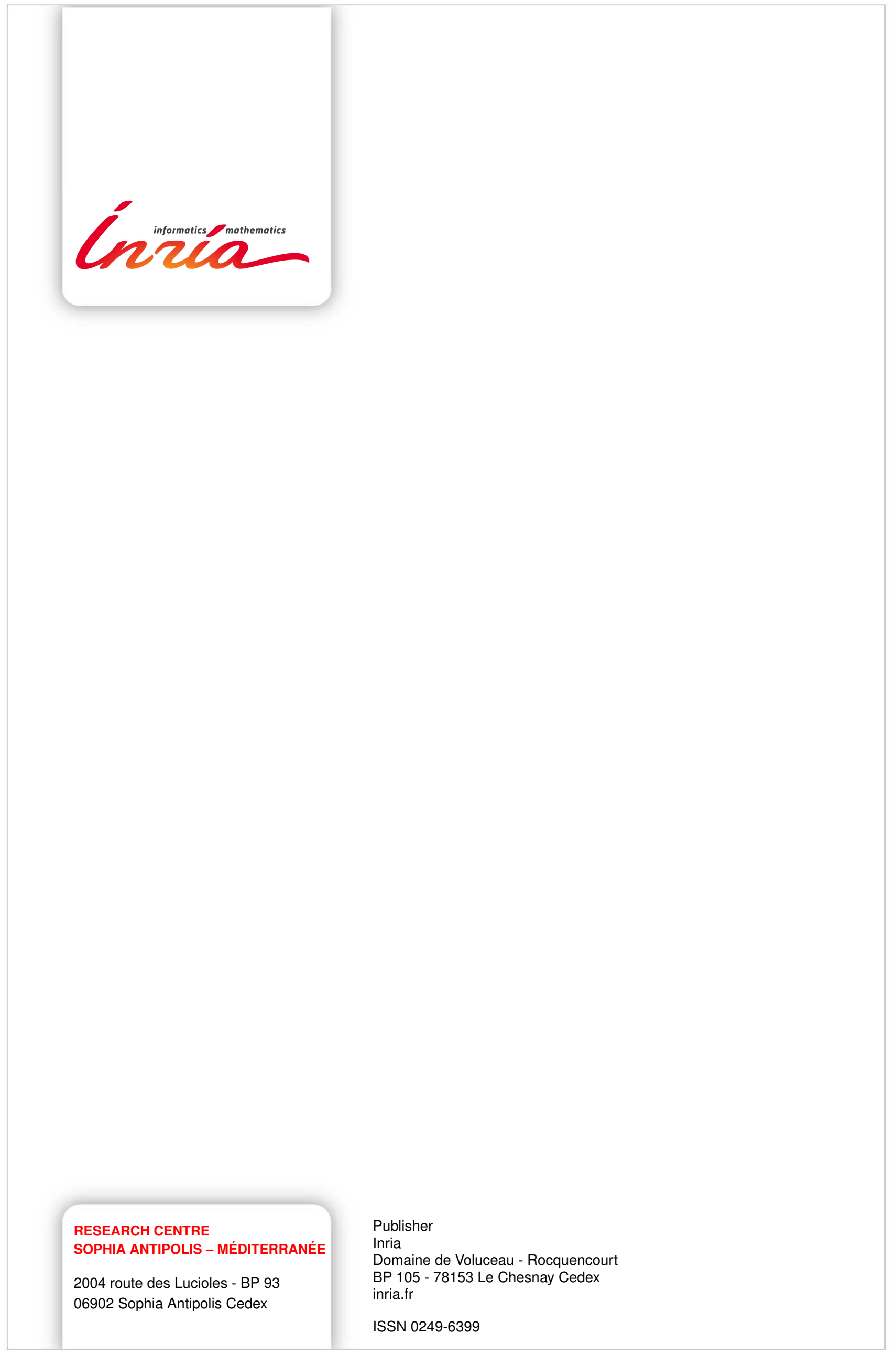

